\title{
Development of Structure Displacement Monitoring System Based on Virtual Instrument Technology
}

\author{
Ying Zhang ${ }^{\text {a }}$ \\ Department of Civil Engineering, Liaoning Water Conservancy Vocational College, Nanyi Road of \\ Hushitai Shenbei New District, Shenyang, LN, China 110122; \\ ayingzi0728@163.com
}

Keywords: Structural health monitoring (SHM); Virtual instrument (VI); LabVIEW; CompactRIO Abstract. With the traditional health monitoring technology as the theoretical basis, a real-time online displacement monitoring system platform was developed based on the NI CompactRIO embedded platform, and modules NI 9219. And the graphical programming software LabVIEW was used to building the background processes and human-computer interface. The main function of the monitoring system developed in the paper is displacement monitoring. It has realized that displacement data of structure acquisition synchronously, signal of the real-time online processing, structure security early warning, and other functions. And a test was hold to verify the stability of the system developed.

\section{Introduction}

The safety of major civil engineering structures such as bridges, power plants, dams, military installations, high-rise buildings, etc. are very important for a country and region. So, it is very essential to the assessment of health status for those important civil engineering structures. The structural health monitoring technology can monitor and forecast the health status of the structures in real time, find the location and degree of structural damage, predict the structure and the remaining life, and provide the basis for the maintenance and maintenance of the structure. That is very important for the protection of people's life and property [1,2].

However, the technology also faces some problems in the course of being used in engineering field. One of problems is the lack of efficient health monitoring systems. The traditional health monitoring equipment is expensive, low efficiency and poor in reconfigurability. As a new direction of the development of modern instruments, virtual instrument has been paid more and more attention $[3,4]$. In particular, the use of virtual instrument for structural health monitoring program can greatly shorten development cycle, reduce development costs. So it becomes the first choice for people to build a modern health monitoring system. The virtual instrument platform based on graphical programming developed by Company NI of American is most popular currently.

In the paper, an efficient and integrated structure displacement monitoring system was developed based on NI Compact RIO and LabVIEW. Using the system, it can realize that displacement data of structure acquisition and processing in real-time, data analysis, storage and query and structure security warning. This lays the foundation for the further application of health monitoring technology in engineering field.

\section{System hardware configuration}

The hardware configuration of the monitoring system proposed in the paper is mainly composed of CompactRIO-9074 embedded development platform, NI 9219 module, and displacement sensors.

CompactRIO-9074 embedded development platform. It is a small and solid control and acquisition system produced by Company NI. Its high-performances can be achieved by reconfigure the I/O based on the FPGA technology. CompactRIO-9074 also includes hot plug industrial-strength I/O modules which have build-in signal conditioner [5]. They can connect sensors directly. The data can be transferred to processor by data-transmission mechanism in the device [6]. The CompactRIO-9074 embedded development platform is shown as Fig. 1. 


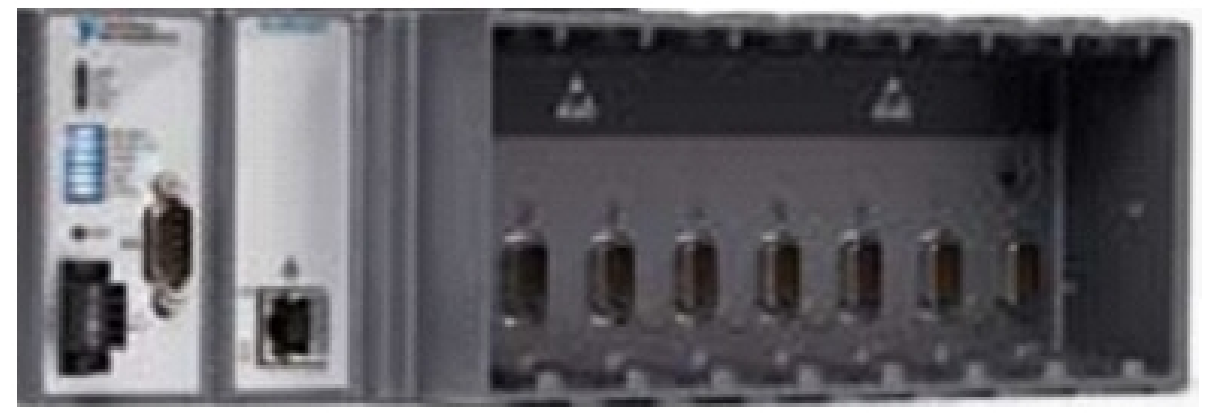

Fig. 1 CompactRIO 9074

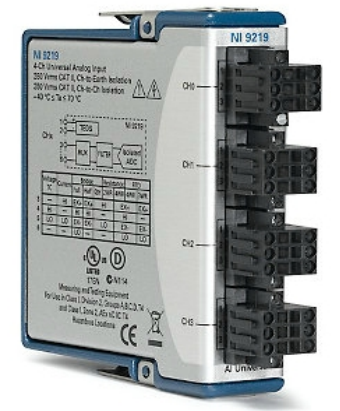

Fig. 2 NI-9219 module

The characters of the device are follows:

(1) Low power consumption and high speed processing capability. That is because the embedded design of platform and the underlying FPGA technology.

(2) Rugged and reliable hardware case. Device is a portable, small size and strong, with the ability to resist the impact of the maximum $50 \mathrm{~g}$ of the acceleration. The device can meet the relative complexity of the working environment, especially the complex and harsh environment.

(3) Modularization. Because of the FPGA technology, the platform can be configured according to the actual needs of a variety of supporting hot plug I/O module, and then realize the needs of multiple applications on a platform, so that the user can quickly complete system prototype design.

(4) Powerful signal processing and analytical skills. The graphical programming software LabVIEW as the development tool of CompactRIO embedded platform, can provide a variety of functions for data processing according to the actual needs of the user.

(5) Short development cycle and low risk. The graphical programming language can make the engineering technical personnel to put their ideas into practice, not only shorten the system development time, but also reduce the system development costs and production technology risk.

I/O modules. NI Company provides a variety of I/O modules for CompactRIO embedded platform. They can complete various digital/analog signals (such as voltage, current, resistance, etc.) input, output and control. They have many forms of interface, supporting a variety of communication protocols and bus. They also can achieve hot swap operation, achieve plug and play purposes, and meet the requirements of engineering applications. The system selects NI-9219 modulus as displacement acquisition card, which is shown as Fig. 2.

\section{Software development of system}

In order to shorten the development cycle of software, and enhance the readability and maintainability of program, the basic idea of modularization and structured programming is adopted in the design process. Software design was based on LabVIEW 10 development environment. In the process of programming, real-Time and FPGA toolkits were used. Software development is divided into two layers: CompactRIO platform programming design and the upper PC programming design.

Program designs for CompactRIO platform. Data acquisition program is realized by LabVIEW and FPGA LabVIEW. FPGA program block diagram is shown as Fig. 3. The sequential structure is used to collect four channel data. In the caching process, the Build Array function was used to build a one-dimensional array, and then write the data into the FIFO DMA buffer, which ensure that the data between the channels do not affect each other.

Program designs for upper PC. The upper computer program is the operation panel and user interface. Therefore, it should have a good visual interface and easy to operate. Its functions include data processing analysis, data storage and help information. The main program VI calls for subroutine VI of each function by event structure nodes. Operator can call the corresponding function by clicking on the functional module button in the main interface. 
Main function modules design. Data processing and analysis module can analyze the signal acquired by time domain, frequency domain and time-frequency domain. The front panel of the program is organized by the Tab controller. Users can view the analysis results by clicking on different options. The program panel is composed of while and Case structure. File management module has the function of data storage and report printing function. Data storage function can complete the original signal record. When it needs to perform data playback or subsequent processing, the original signal record file can be read. In the help module, users can click on the "help information" button to get instructions.

\section{System test}

In order to test the stability and reliability of the monitoring system proposed in this paper, a three layer steel frame model was selected as the test object. The model size is $600 \mathrm{~mm} \times 400 \mathrm{~mm} \times 1500 \mathrm{~mm}$. Shaking table was used to carry out seismic wave action. At the same time, the traditional data acquisition devices were used to collect the same part of the data signals as the test data comparison. The test object and devices are shown in Fig. 3. In the test, two YHD-20 displacement sensors were fixed on the model used for monitoring displacement of test object and table surface. They are shown in Fig. 4 and Fig. 5.

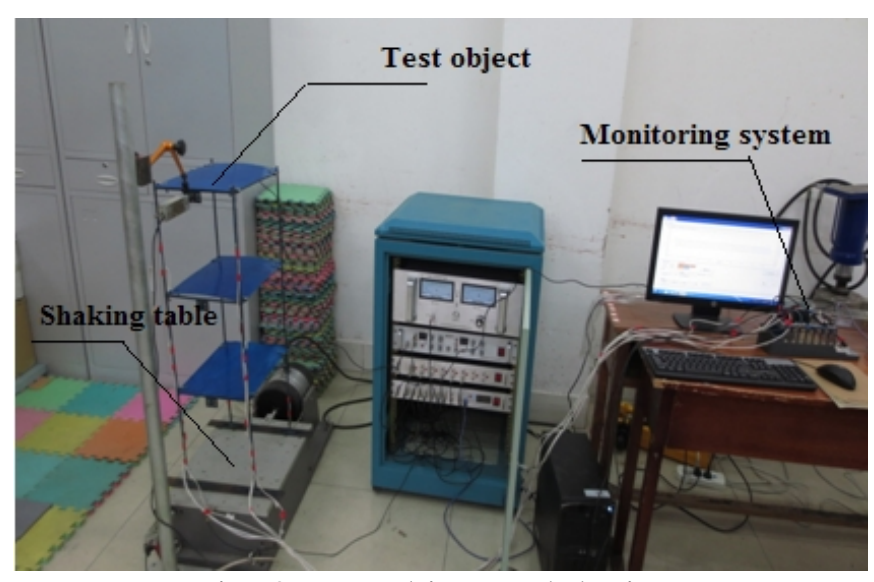

Fig. 3 Test object and devices

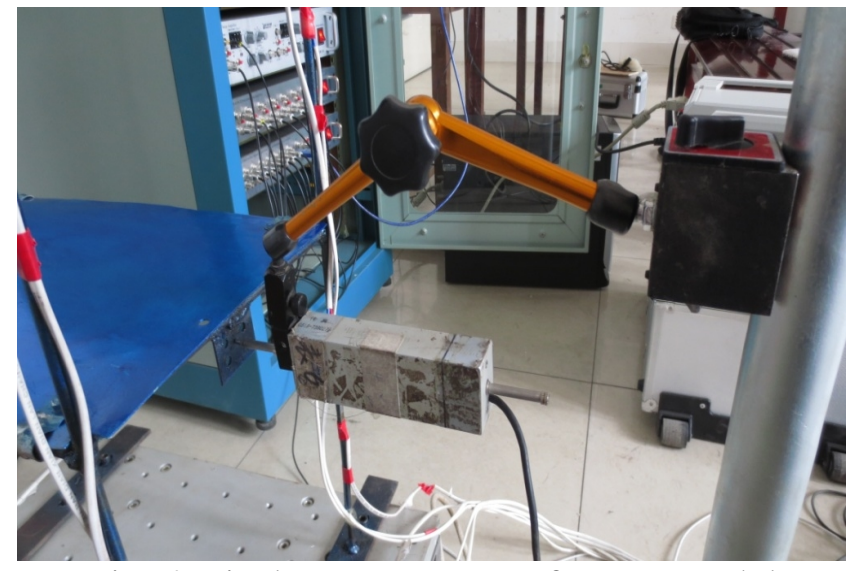

Fig. 4 Displacement sensor for test model

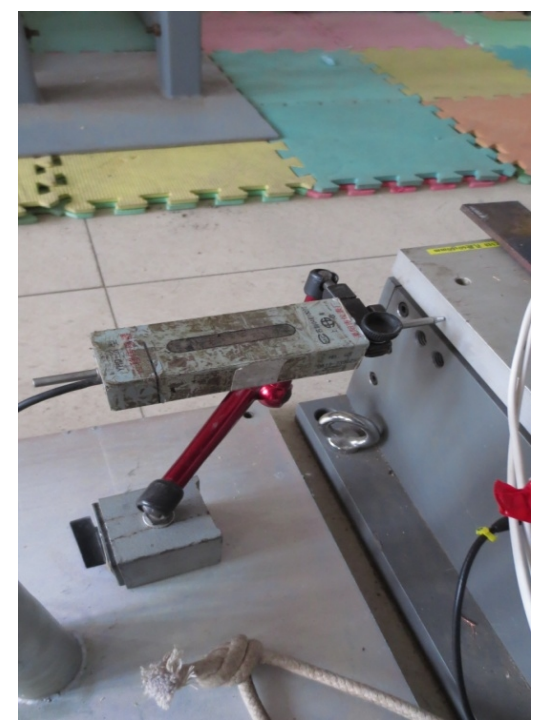

Fig. 5 Displacement sensor for test model

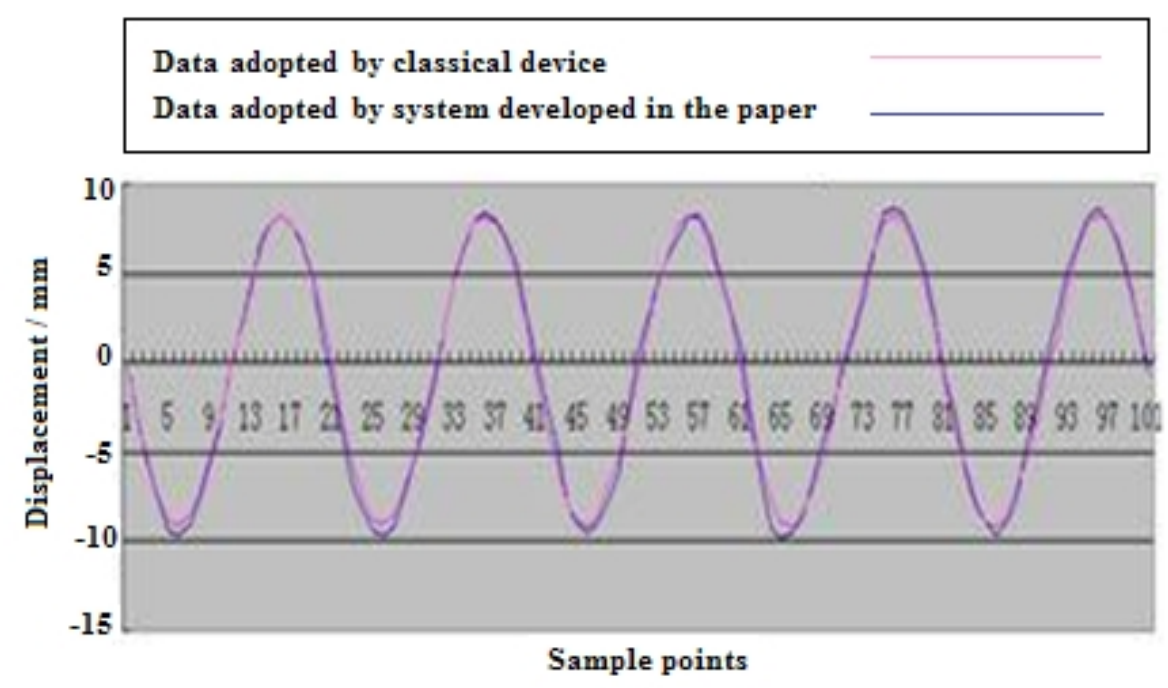

Fig. 6 Displacement sensor for test model

The displacement data of the test model are acquired through the development of the system and the traditional data acquisition system. And then, two groups of data were compared in order to investigate 
whether the two had good agreement. One group contrastive data is shown in Fig. 6. Red curve is the data adopted by classical device, and the blue one is by system proposed in the paper. From the figure it can be seen that two curves almost coincidence. It means that the system developed in the paper is reliable.

\section{Conclusions}

In this paper, a structure displacement monitoring system based on LabVIEW development environment and CompactRIO hardware platform was developed by using virtual instrument technology. Compared with the traditional method, the system developed in the paper has the advantages of high reliability, simple structure, can adapt to the complex environment. At the same time, due to the flexible and robust of RIO compact platform, it can be fixed on the any situation of the test object in accordance with needs. Virtual instrument technology was used during the development of the system, which greatly enhance the integration and portability of the system. That has laid a foundation for engineering applications of the system. Graphical programming software LabVIEW and reusable embedded platform CompactRIO also can shorten the development cycle and cost of the system. The work of this paper is to lay a good foundation, realizing multi physical quantity structural health monitoring system in the future.

\section{Acknowledgements}

This work was financially supported by Liaoning Provincial Committee of Education Research Fund Project China (L2013541).

\section{References}

[1] Zhu Hongping, Yu Jing, Zhang Junbing. A summary review and advantages of vibration-based damage identification methods in structural health monitoring. Engineering mechanis, Vol.28(2011), p1

[2] Li Hongnan, Li Dongsheng. Safety assessment, health monitoring and damage diagnosis for structures in civil engineering. Earthquake engineering and engineering vibration, Vol.22(2004), p82

[3] Jiang Qi, Zhang Fang. The dynamic strain test system based on LabVIEW. Research and development, Vol.29(2010), p40

[4] Shen Guanlin. The new development of strain electrical measurement and sensor technology and application. Chinese test, Vol.37(2011), p87

[5] Gao Zhanfeng, Du Yanliang, Liu Yuhong. Remote bridge health data acquisition system based on virtual instrument. Microelectronics and computer, Vol.24(2007), p102

[6] Yu Ling, Zhang Junhua, Chan T H T. A LabVIEW based data acquisition system for bridge health monitoring. Journal of Jinan University, Vol.30(2009), p465 